\title{
Analytical Calculation of Magnetic Field in Surface-Inset Permanent Magnet Motors
}

\author{
Linni Jian, K. T. Chau, Yu Gong, Chuang Yu, and Wenlong Li \\ Department of Electrical and Electronic Engineering, The University of Hong Kong, Hong Kong, China
}

\begin{abstract}
This paper presents an analytical approach to calculate the magnetic field distribution in surface-inset permanent magnet motors. A series-slot analytical model is newly proposed to describe the magnetic field behavior by a set of partial differential equations in terms of scalar magnetic potentials. The field solutions are determined by considering the boundary constraints. Then, the cogging torque characteristic is deduced by calculating the Maxwell tensors. The accuracy of the proposed analytical calculation is verified by comparing the field distribution and cogging torque results with those obtained from the finite element method.
\end{abstract}

Index Terms-Analytical calculation, finite element method, magnetic field distribution, surface-inset permanent magnet motors.

\section{INTRODUCTION}

B $\mathrm{Y}$ incorporating the merits of the field-regulated reluctance machine and the surface-mounted permanent magnet (SMPM) machine, the surface-inset PM motor (SIPM) [1] has been proposed to meet the demands arising from advanced electric traction [2]. Fig. 1 shows a 6-pole 4-phase SIPM motor. The PMs are radially magnetized and inserted in the sunken space of the rotor. Since the PM has a relative permeability similar to that of air, the q-axis inductance is much higher than the d-axis inductance, hence producing the reluctance torque [3]. Moreover, it can offer some distinct advantages: namely the simple and robust rotor structure, high torque density, easy-to-achieve flux-weakening operation and potentiality of fully sensorless operation.

As well known, the detailed knowledge of the field distributions in the airgap is vitally important for predicting and optimizing the performance of PM motors. Although the field calculation of various PM motors can be accurately performed by using numerical approaches such as the finite element method (FEM), they can provide neither closed-form solution nor physical insight for designers. Therefore, the development of analytical approaches has attracted more and more attention [4]-[6]. However, all relevant publications were focused on the SMPM motors in which the single-slot model was employed in the derivation. The purpose of this paper is to propose an analytical approach to calculate the magnetic field distribution in SIPM motors. Unlike the approaches for the SMPM motors, the proposed approach will take into account the field in the region of PMs. Also, the series-slot model will be newly engaged to improve the accuracy for calculating the magnetic flux density and cogging torque. The validity of the proposed analytical approach will be verified by comparing the calculation results with those obtained from the FEM.

\section{ANALYTICAL MODEL}

Fig. 1 depicts a typical 4-phase SIPM motor, which can also be configured as 3 or 5 phases. Fig. 2 shows its series-slot model with a pole-pair pitch. For simplicity, the depth of the slots and the permeability of the iron yokes are assumed to be infinite. The widths of the PMs, salient poles, slots and teeth are denoted

Manuscript received March 01, 2009; revised April 20, 2009. Current version published September 18, 2009. Corresponding author: L. Jian (e-mail: lnjian@eee.hku.hk).

Digital Object Identifier 10.1109/TMAG.2009.2024891

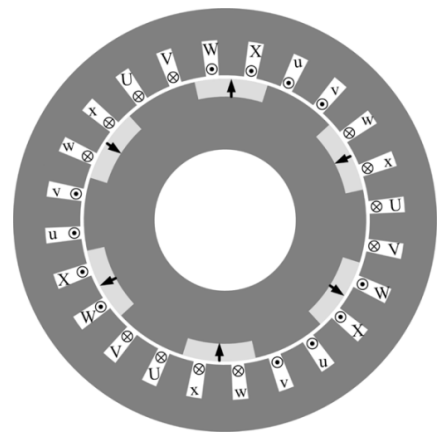

Fig. 1. Surface-inset permanent magnet motor.

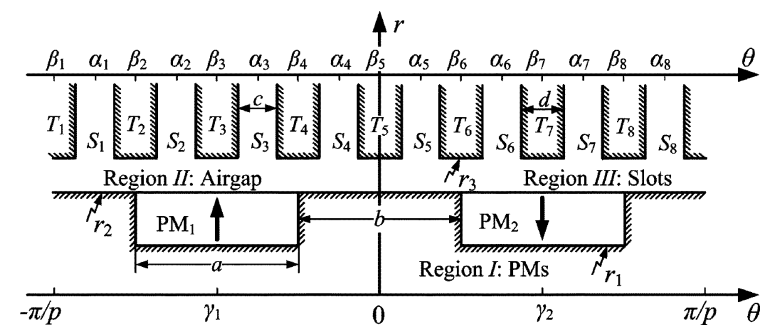

Fig. 2. Analytical model.

by $a, b, c$, and $d$, respectively. Also, $p$ is the pole-pair number, $r_{1}, r_{2}$, and $r_{3}$ are the inside radius of the PMs, radius of the rotor and inside radius of the stator, respectively. The $\theta$-axis coordinates of the central lines of the $i$ th slot $S_{i}$, the $i$ th tooth $T_{i}$, and the $j$ th PM are denoted by $\alpha_{i}, \beta_{i}$, and $\gamma_{j}$, respectively.

The calculation region can be classified into three parts: PMs (Region I), airgap (Region II), and slots (Region III). In various regions, the flux density $\vec{B}$ and field intensity $\vec{H}$ are expressed as

$$
\begin{aligned}
& \text { In Region I: } \vec{B}=\mu_{0} \mu_{r} \vec{H}+\mu_{0} \vec{M} \\
& \text { In Region II, III: } \vec{B}=\mu_{0} \vec{H}
\end{aligned}
$$

where $\mu_{r}$ is the relative recoil permeability, $\vec{M}$ is the residual magnetization vector. By employing the scalar magnetic potential $\varphi$, the field behavior can be governed by a set of 2-rank partial differential equations:

$$
\begin{array}{ll}
\text { In Region I : } & \nabla^{2} \varphi^{I}(r, \theta)=\operatorname{div}(\vec{M}) / \mu_{r} \\
\text { In Region II : } & \nabla^{2} \varphi^{I I}(r, \theta)=0 \\
\text { In Region III : } & \nabla^{2} \varphi^{I I I}(r, \theta)=0 .
\end{array}
$$


The corresponding boundary conditions can be defined as

$$
\begin{aligned}
& r=r_{1} \& \theta \in\left[\gamma_{j}-a / 2, \gamma_{j}+a / 2\right]: \varphi_{j}^{\mathrm{I}}\left(r_{1}, \theta\right)=0 \\
& \theta=\gamma_{j} \pm a / 2 \quad r \in\left[r_{1}, r_{2}\right]: \varphi_{j}^{\mathrm{I}}\left(r, \gamma_{j}-a / 2\right)=0 \\
& r=r_{2} \& \theta \in[-b / 2, b / 2]: \varphi^{\mathrm{II}}\left(r_{2}, \theta\right)=0 \\
& r=r_{2} \& \theta \in[-\pi / p,-\pi / p+b / 2]: \varphi^{\mathrm{II}}\left(r_{2}, \theta\right)=0 \\
& r=r_{2} \& \theta \in[\pi / p-b / 2, \pi / p]: \varphi^{\mathrm{II}}\left(r_{2}, \theta\right)=0 \\
& r=r_{2} \& \theta \in\left[\gamma_{j}-a / 2, \gamma_{j}+a / 2\right]: \\
& \varphi_{j}^{\mathrm{I}}\left(r_{2}, \theta\right)=\varphi^{\mathrm{II}}\left(r_{2}, \theta\right) ; \\
& \left.\mu_{r}\left(\partial \varphi^{\mathrm{II}} / \partial r\right)\right|_{r=r_{2}}=\left.\mu_{r}\left(\partial \varphi_{j}^{\mathrm{I}} / \partial r\right)\right|_{r=r_{2}}-M_{\mathrm{rj}} \\
& r=r_{3} \& \theta \in\left[\beta_{i}-c / 2, \beta_{i}+c / 2\right](i=1-8): \\
& \varphi^{\mathrm{II}}\left(r_{3}, \theta\right)=0 \\
& r=r_{3} \& \theta \in\left[\alpha_{i}-c / 2, \alpha_{i}+c / 2\right](i=1-8): \\
& \varphi^{\mathrm{II}}\left(r_{3}, \theta\right)=\varphi_{i}^{\mathrm{III}}\left(r_{3}, \theta\right) ; \\
& \left.\left(\partial \varphi^{\mathrm{II}} / \partial r\right)\right|_{r=r_{3}}=\left.\left(\partial \varphi_{i}^{\mathrm{III}} / \partial r\right)\right|_{r=r_{3}} \\
& \theta=\alpha_{i} \pm c / 2 \& r \in\left[r_{3}, \infty\right](i=1-8): \\
& \quad \varphi_{i}^{\mathrm{III}}\left(r, \alpha_{i} \pm c / 2\right)=0 \\
& r=+\infty \& \theta \in\left[\alpha_{i}-c / 2, \alpha_{i}+c / 2\right](i=1-8): \\
& \varphi_{i}^{\mathrm{III}}\left(r_{3}, \theta\right)=0
\end{aligned}
$$

where $\varphi_{j}^{\mathrm{I}}(j=1,2)$ is the scalar potential in the $j$ th PM, and $\varphi_{i}^{\mathrm{III}}(i=1-8)$ is the scalar potential in the $i$ th slot.

\section{FIELD SOLUTION}

\section{A. Field Distribution in Airgap}

The scalar potentials in Region II are governed by the Laplace's equation given in (4). By separating the variables $r$ and $\theta$, the general solution can be expressed as

$$
\varphi^{\mathrm{II}}=\sum_{n=1}^{\infty}\left[\begin{array}{c}
\left(D_{n} r^{n p}+E_{n} r^{-n p}\right) \cos n p \theta \\
+\left(F_{n} r^{n p}+G_{n} r^{-n p}\right) \sin n p \theta
\end{array}\right]+D_{0} \ln r+E_{0}
$$

where $D_{n}, E_{n}, F_{n}, G_{n}, D_{0}$, and $E_{0}$ are the Fourier coefficients to be determined.

\section{B. Field Distribution in Permanent Magnets}

The scalar potential in the $j$ th PM is governed by the Poissonian equation given in (3). According to the superposition law, its general solution is the sum of the solution of the corresponding Laplace's equation and one special solution of its own.

Firstly, the general solution of the corresponding Laplace's equation is considered. In order to satisfy the boundary conditions given by (7), by using the method of separating variables, the general solution is given by

$$
\varphi_{1 j}^{\mathrm{I}}=\sum_{m=1}^{\infty}\left[\left(A_{j m} r^{\lambda_{m a}}+B_{j m} r^{-\lambda_{m a}}\right) \sin \lambda_{m a}\left(\theta-\gamma_{j}+a / 2\right)\right]
$$

where $\lambda_{m a}=m \pi / a$, and $A_{j m}$ and $B_{j m}$ are the Fourier coefficients to be determined.

Secondly, the special solution of the Poissonian equation is taken into account. In polar coordinates, the residual magnetization can be expressed as

$$
\vec{M}_{j}=M_{j r} \vec{r}+M_{j \theta} \vec{\theta}
$$

where $M_{j \theta}=0, M_{j r}=M_{r}$ when $j=1$, and $M_{j r}=-M_{r}$ when $j=2$. From (3) and (20), it yields

$$
\operatorname{div}\left(\vec{M}_{j}\right) / \mu_{r}=M_{j r} /\left(\mu_{r} r\right) .
$$

By applying the Fourier series expansion over $\left[\gamma_{j}-a / 2, \gamma_{j}+\right.$ $a / 2$ ], it yields

$$
\begin{aligned}
& \frac{\operatorname{div}\left(\vec{M}_{j}\right)}{\mu_{r}}=\sum_{m=1}^{\infty}\left[\frac{2\left(1-(-1)^{m}\right) M_{j r}}{a r \mu_{r} \lambda_{m a}}\right. \\
& \left.\times \sin \lambda_{m a}\left(\theta-\gamma_{j}+a / 2\right)\right] .
\end{aligned}
$$

Thus, the special solution of (3) is given by

$$
\varphi_{2 j}^{\mathrm{I}}=\sum_{m=1}^{\infty}\left[\frac{2 r\left(1-(-1)^{m}\right) M_{j r}}{a \mu_{r} \lambda_{m a}\left(1-\lambda_{m a}^{2}\right)} \sin \lambda_{m a}\left(\theta-\gamma_{j}+a / 2\right)\right] .
$$

Finally, the general solution of the scalar potential in Region $I$ can be obtained as

$$
\begin{aligned}
\varphi_{j}^{\mathrm{I}}=\sum_{m=1}^{\infty}\left[\left(\begin{array}{c}
A_{j m} r^{\lambda_{m a}}+B_{j m} r^{-\lambda_{m a}} \\
+\frac{2 r\left(1-(-1)^{m}\right) M_{j r}}{a \mu_{r} \lambda_{m a}\left(1-\lambda_{m}^{2}\right)}
\end{array}\right)\right. \\
\left.\times \sin \lambda_{m a}\left(\theta-\gamma_{j}+a / 2\right)\right] .
\end{aligned}
$$

\section{Field Distribution in Slots}

The scalar potential in the $i$ th slot is governed by the Laplace's equation given in (5). Considering the boundary conditions given in (16) and (17), by using the method of separating variables, its general solution can be obtained as

$$
\varphi_{i}^{\mathrm{III}}=\sum_{m=1}^{\infty}\left[C_{i m} r^{-\lambda_{m c}} \sin \lambda_{m c}\left(\theta-\alpha_{i}+c / 2\right)\right]
$$

where $\lambda_{m c}=m \pi / c$, and $C_{i m}$ are the Fourier coefficients to be determined.

\section{Boundary Conditions}

Firstly, on the inside surface of the PMs $\left(r=r_{1}\right)$, from (6) and (24), it yields

$$
A_{j m} r_{1}^{\lambda_{m a}}+B_{j m} r_{1}^{-\lambda_{m a}}+\frac{2\left(1-(-1)^{m}\right) r_{1} M_{j r}}{a \mu_{r} \lambda_{m a}\left(1-\lambda_{m a}^{2}\right)}=0 .
$$

Secondly, on the surface of the rotor PMs $\left(r=r_{2}\right)$, from (8)-(11), the scalar potential on this surface can be expanded into Fourier series over $[-\pi / p, \pi / p]$ as given by

$$
\begin{aligned}
& \varphi^{\mathrm{II}}\left(r_{2}, \theta\right)=d_{0} / 2+\sum_{n=1}^{\infty}\left[d_{n} \cos n p \theta+f_{n} \sin n p \theta\right] \\
& d_{0}=\frac{p}{\pi} \int_{-\pi / p}^{\pi / p} \varphi^{\mathrm{II}}\left(r_{2}, \theta\right) d \theta \\
& =\frac{p}{\pi} \sum_{j=1}^{2} \sum_{m=1}^{\infty}\left[\left(1-(-1)^{m}\right)\left(A_{j m} r_{2}^{\lambda_{m a}}+B_{j m} r_{2}^{-\lambda_{m a}}\right) / \lambda_{m a}\right]
\end{aligned}
$$




$$
\begin{aligned}
& d_{n}=\frac{p}{\pi} \int_{-\pi / p}^{\pi / p} \varphi^{\mathrm{II}}\left(r_{2}, \theta\right) \cos n p \theta d \theta \\
& =\frac{p}{\pi} \sum_{j=1}^{2} \sum_{m=1}^{\infty} \tau(n, m, j, a, \gamma)\left(\begin{array}{c}
A_{j m} r_{2}^{\lambda_{m a}}+B_{j m} r_{2}^{-\lambda_{m a}} \\
+\frac{2 r_{2}\left(1-(-1)^{m}\right) M_{j r}}{a \lambda_{m a} \mu_{r}\left(1-\lambda_{m a}^{2}\right)}
\end{array}\right) \\
& f_{n}=\frac{p}{\pi} \int_{-\pi / p}^{\pi / p} \varphi^{\mathrm{II}}\left(r_{2}, \theta\right) \sin n p \theta d \theta \\
& =\frac{1}{\pi} \sum_{j=1}^{2} \sum_{m=1}^{\infty} \omega(n, m, j, a, \gamma)\left(\begin{array}{c}
A_{j m} r_{2}^{\lambda_{m a}}+B_{j m} r_{2}^{-\lambda_{m a}} \\
+\frac{2 r_{2}\left(1-(-1)^{m}\right) M_{j r}}{a \lambda_{m a} \mu_{r}\left(1-\lambda_{m a}^{2}\right)}
\end{array}\right)
\end{aligned}
$$

where $\tau(\cdot)$ and $\omega(\cdot)$ are defined by (31) and (32), shown at the bottom of the page. Thus, from (18) and (27), it yields

$$
\begin{aligned}
d_{0} / 2 & =D_{0} \ln r_{2}+E_{0} \\
d_{n} & =D_{n} r_{2}^{n p}+E_{n} r_{2}^{-n p} \\
f_{n} & =F_{n} r_{2}^{n p}+G_{n} r_{2}^{-n p} .
\end{aligned}
$$

Moreover, since (12) denotes the continuity of the flux density through this surface, the integration of both sides of (12) with a factor of $\sin \lambda_{m a}\left(\theta-\gamma_{j}+a / 2\right)$ yields

$$
\begin{array}{r}
\int_{r_{j}-a / 2}^{r_{j}+a / 2}\left(\left.\mu_{r}\left(\partial \varphi_{j}^{\mathrm{I}} / \partial r\right)\right|_{r=r_{2}}+M_{r j}\right) \sin \lambda_{m a}\left(\theta-\gamma_{j}+a / 2\right) d \theta \\
=\left.\int_{r_{j}-a / 2}^{r_{j}+a / 2} \mu_{r}\left(\partial \varphi^{\mathrm{II}} / \partial r\right)\right|_{r=r_{2}} \sin \lambda_{m a}\left(\theta-\gamma_{j}+a / 2\right) d \theta .
\end{array}
$$

Substituting (18) and (27) into (36), it yields

$$
\begin{aligned}
& \frac{m \pi\left(A_{j m} r_{2}^{\lambda_{m a}}-B_{j m} r_{2}^{-\lambda_{m a}}\right)}{2} \\
& +\frac{r_{2} M_{j r}\left(1+\mu_{r}\left(1-\lambda_{m a}^{2}\right)\right)\left(1-(-1)^{m}\right)}{\mu_{r} \lambda_{m a}\left(1-\lambda_{m a}^{2}\right)} \\
& \quad=\sum_{n=1}^{\infty} n p\left[\begin{array}{c}
\left(D_{n} r_{2}^{n p}-E_{n} r_{2}^{-n p}\right) \tau(n, m, j, a, \gamma) \\
+\left(F_{n} r_{2}^{n p}-G_{n} r_{2}^{-n p}\right) \omega(n, m, j, a, \gamma)
\end{array}\right] \\
& \quad+\frac{D_{0}\left(1-(-1)^{m}\right)}{\lambda_{m a}} .
\end{aligned}
$$

Thirdly, on the inside surface of the stator $\left(r=r_{3}\right)$, from (13) and (14), the scalar potential on this surface can be expanded into Fourier series over $[-\pi / p, \pi / p]$ as given by

$$
\begin{aligned}
\varphi^{\mathrm{II}}\left(r_{3}, \theta\right) & =e_{0} / 2+\sum_{n=1}^{\infty}\left[e_{n} \cos n p \theta+g_{n} \sin n p \theta\right] \\
e_{0} & =\frac{p}{\pi} \int_{-\pi / p}^{\pi / p} \varphi^{\mathrm{II}}\left(r_{3}, \theta\right) d \theta \\
& =\frac{p}{\pi} \sum_{i=1}^{8} \sum_{m=1}^{\infty}\left[\left(1-(-1)^{m}\right)\left(C_{i m} r_{3}^{-\lambda_{m c}}\right) / \lambda_{m c}\right] \\
e_{n} & =\frac{p}{\pi} \int_{-\pi / p}^{\pi / p} \varphi^{\mathrm{II}}\left(r_{3}, \theta\right) \cos n p \theta d \theta \\
& =\frac{p}{\pi} \sum_{i=1}^{8} \sum_{m=1}^{\infty}\left[\tau(n, m, i, c, \alpha)\left(C_{i m} r_{3}^{-\lambda_{m c}}\right)\right] \\
g_{n} & =\frac{p}{\pi} \int_{-\pi / p}^{\pi / p} \varphi^{\mathrm{II}}\left(r_{3}, \theta\right) \sin n p \theta d \theta \\
& =\frac{p}{\pi} \sum_{i=1}^{8} \sum_{m=1}^{\infty}\left[\omega(n, m, i, c, \alpha)\left(C_{i m} r_{3}^{-\lambda_{m c}}\right)\right]
\end{aligned}
$$

Thus, from (18) and (38), it yields

$$
\begin{aligned}
e_{0} / 2 & =D_{0} \ln r_{3}+E_{0} \\
e_{n} & =D_{n} r_{3}^{n p}+E_{n} r_{3}^{-n p} \\
g_{n} & =F_{n} r_{3}^{n p}+G_{n} r_{3}^{-n p}
\end{aligned}
$$

Moreover, the integration of both two sides of (15) with a factor of $\sin \lambda_{m c}\left(\theta-\alpha_{j}+c / 2\right)$ yields

$$
\begin{aligned}
& \left.\int_{\alpha_{i}-c / 2}^{\alpha_{i}+c / 2}\left(\partial \varphi_{i}^{\mathrm{III}} / \partial r\right)\right|_{r=r_{3}} \sin \lambda_{m c}\left(\theta-\alpha_{i}+c / 2\right) d \theta \\
& =\left.\int_{\alpha_{i}-c / 2}^{\alpha_{i}+c / 2}\left(\partial \varphi^{\mathrm{II}} / \partial r\right)\right|_{r=r_{3}} \sin \lambda_{m c}\left(\theta-\alpha_{i}+c / 2\right) d \theta .
\end{aligned}
$$

Substituting (18) and (27) into (45), it yields

$$
\begin{aligned}
- & m \pi C_{i m} r_{3}^{-\lambda_{m c}} / 2 \\
= & \sum_{n=1}^{\infty} n p\left[\begin{array}{c}
\left(D_{n} r_{3}^{n p}-E_{n} r_{3}^{-n p}\right) \tau(n, m, i, c, \alpha) \\
+\left(F_{n} r_{3}^{n p}-G_{n} r_{3}^{-n p}\right) \omega(n, m, i, c, \alpha)
\end{array}\right] \\
+ & \frac{D_{0}\left(1-(-1)^{m}\right)}{\lambda_{m c}} .
\end{aligned}
$$

$$
\begin{gathered}
\tau(n, m, k, z, \Gamma)= \begin{cases}\frac{\lambda_{m z}\left((-1)^{m} \cos n p\left(\Gamma_{k}+z / 2\right)-\cos n p\left(\Gamma_{k}-z / 2\right)\right)}{(n p)^{2}-\lambda_{m x}^{2}} & n p \neq \lambda_{m z} \\
\frac{\cos n p\left(\Gamma_{k}-z / 2\right)-(-1)^{m} \cos n p\left(\Gamma_{k}+z / 2\right)}{2\left(n p+\lambda_{m z}\right)}-\frac{z \sin \lambda_{m z}\left(\Gamma_{k}-z / 2\right)}{2} & n p=\lambda_{m z}\end{cases} \\
\omega(n, m, k, z, \Gamma)=\left\{\begin{array}{ll}
\frac{\lambda_{m z}\left((-1)^{m} \sin n p\left(\Gamma_{k}+z / 2\right)-\sin n p\left(\Gamma_{k}-z / 2\right)\right)}{(n p)^{2}-\lambda_{m z}^{2}} & n p \neq \lambda_{m z} \\
\frac{\sin n p\left(\Gamma_{k}-z / 2\right)-(-1)^{m} \sin n p\left(\Gamma_{k}+z / 2\right)}{2\left(n p+\lambda_{m z}\right)}+\frac{z \cos \lambda_{m z}\left(\Gamma_{k}-z / 2\right)}{2} & n p=\lambda_{m z}
\end{array} .\right.
\end{gathered}
$$


TABLE I

MOTOR SPECIFICATIONS

\begin{tabular}{ll}
\hline \hline Rated power $(\mathrm{W})$ & 800 \\
Rated voltage $(\mathrm{V})$ & 48 \\
Rated speed $(\mathrm{rpm})$ & 1000 \\
Stator outside radius $(\mathrm{mm})$ & 60 \\
Stator slot width and depth $($ degree $\times \mathrm{mm})$ & $5 \times 10$ \\
Airgap length $(\mathrm{mm})$ & 1 \\
Axial core length $(\mathrm{mm})$ & 50 \\
Rotor outside radius $(\mathrm{mm})$ & 40 \\
Rotor inside radius $(\mathrm{mm})$ & 20 \\
PM pole width and depth $($ degree $\times \mathrm{mm})$ & $30 \times 5$ \\
PM remanence $(\mathrm{T})$ & 1.3 \\
\hline \hline
\end{tabular}

Therefore, based on the aforementioned boundary conditions, the unknown quantities $A_{j m}, B_{j m}, C_{i m}, D_{n}, E_{n}, F_{n}, G_{n}, D_{0}$, and $E_{0}$ can be determined by (26), (33)-(35), (37), (42)-(44), and (46). Hence, the flux density distributions can be deduced from the scalar potential by using

$$
\begin{aligned}
& B_{r}=-\mu_{0}(\partial \varphi / \partial r) \\
& B_{\theta}=-\mu_{0}(\partial \varphi / \partial \theta) / r .
\end{aligned}
$$

Consequently, the torque developed on the rotor can be obtained by calculating the Maxwell stress tensors in the air gap

$$
T_{m}=L_{\mathrm{ef}} R_{m}^{2} \int_{0}^{2 \pi} B_{r} B_{\theta} d \theta / \mu_{0}
$$

where $L_{\text {ef }}$ is the effective axial length, $R_{m}$ is the radius of the central line of the air gap, $B_{r}$ and $B_{\theta}$ are the radial and tangential flux densities in the air gap, respectively.

\section{CAlculation Results}

In order to assess the validity of the proposed analytical method, the corresponding results obtained by FEM are provided for comparison. The specifications of the motor are listed in Table I.

By using (18), (26), (33)-(35), (37), (42)-(44), and (46), the scalar potential waveforms along the inside and outside surfaces of the air gap are calculated as depicted in Fig. 3(a). Hence, by using (47) and (48), the corresponding radial and tangential flux density waveforms are deduced as plotted in Fig. 3(b) and (c), respectively. These flux density waveforms are also deduced by using the FEM, and plotted together with the analytical waveforms. It can be seen that the analytical results closely agree with the FEM results. Additionally, by using (49), the cogging torque waveform is calculated and also compared with that obtained from the FEM as shown in Fig. 3(d). It further confirms that the analytical result agrees well with the FEM result. Most importantly, the proposed analytical method can analyze the static characteristics of SIPM motors at a glance, and provide physical insight for designers to perform optimization.

\section{CONCLUSION}

In this paper, an analytical approach to calculate the magnetic field distribution in SIPM motors has been proposed and verified. Also, by calculating the Maxwell stress in the air gap, the cogging torque can be deduced. The analytical results agree very well with that obtained from the FEM, which enables the pro-

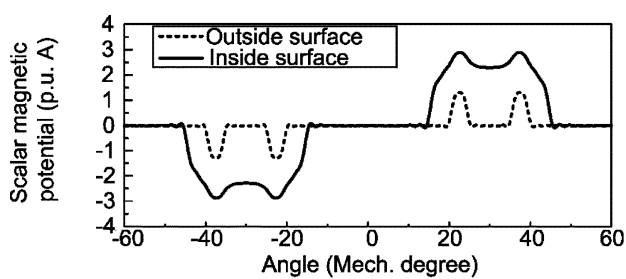

(a)

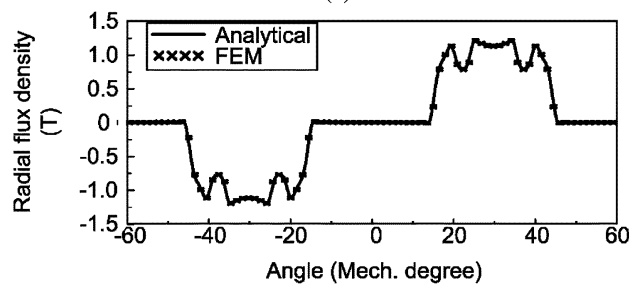

(b)

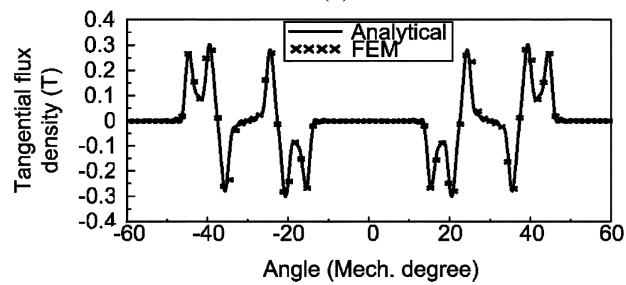

(c)

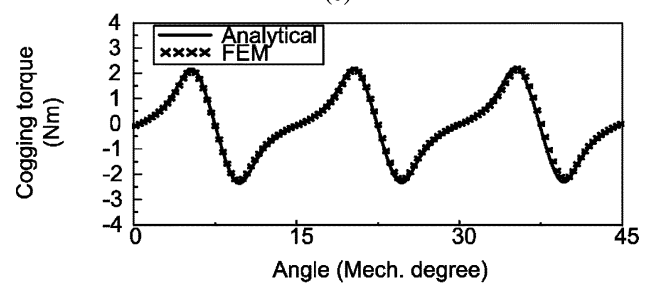

(d)

Fig. 3. Calculation results. (a) Scalar magnetic potential in air gap. (b) Radial flux density. (c) Tangential flux density. (d) Cogging torque.

posed analytical approach to be a useful tool for design and optimization of SIPM motors.

\section{ACKNOWLEDGMENT}

This work was supported by the Research Grants Council, Hong Kong Special Administrative Region, China, under Grant HKU 7105/07E.

\section{REFERENCES}

[1] J. Gan, K. T. Chau, C. C. Chan, and J. Z. Jiang, "A new surfaceinset, permanent-magnet, brushless dc motor drive for electric vehicles," IEEE Trans. Magn., vol. 36, no. 5, pp. 3810-3818, Sep. 2000.

[2] K. T. Chau and C. C. Chan, "Emerging energy-efficient technologies for hybrid electric vehicles," Proc. IEEE, vol. 95, no. 4, pp. 821-835, Apr. 2007.

[3] K. T. Chau, C. C. Chan, and C. Liu, "Overview of permanent magnet brushless drives for electric and hybrid electric vehicles," IEEE Trans. Ind. Electron., vol. 55, no. 6, pp. 2246-2257, Jun. 2008.

[4] Z. Q. Zhu and D. Howe, "Instantaneous magnetic field distribution in brushless permanent magnet dc motors, Part III: Effect of stator slotting," IEEE Trans. Magn., vol. 29, no. 1, pp. 143-151, Jan. 1993.

[5] D. Zarko, D. Ban, and T. A. Lipo, "Analytical calculation of magnetic field distribution in the slotted air gap of a surface permanent-magnet motor using complex relative air-gap permeance," IEEE Trans. Magn., vol. 42, no. 7, pp. 1828-1837, Jul. 2006.

[6] Z. J. Liu and J. T. Li, "Analytical solution of air-gap field in permanentmagnet motors taking into account the effect of pole transition over slots," IEEE Trans. Magn., vol. 43, no. 10, pp. 3872-3883, Oct. 2007. 